\title{
Impact of the ion-cyclotron-resonance location on poloidal asymmetries of impurity density in ICRF-heated rotating plasma
}

\author{
R. Bilato ${ }^{1}$, T. Odstrcil ${ }^{1}$, F.J. Casson $^{2}$, C. Angioni ${ }^{1}$, \\ M. Brambilla ${ }^{1}$, Ye. O. Kazakov ${ }^{3}$, E. Poli ${ }^{1}$ \\ ${ }^{1}$ Max Planck Institute for Plasma Physics, Garching, Germany \\ ${ }^{2}$ CCFE, Culham Science Centre, Abingdon, Oxon, OX14 3DB, UK \\ ${ }^{3}$ Laboratory for Plasma Physics, LPP-ERM/KMS, Brussels, Belgium \\ E-mail: roberto(dot)bilato (at)ipp(dot)mpg(dot)de
}

\begin{abstract}
The recently proposed set of zeroth-order moment equations to model poloidal density asymmetries induced by temperature anisotropies in rotating tokamak plasmas (R. Bilato et al., Nuclear Fusion 54 (2014) 072003) is here extended to account for the effects of the localization of the ion cyclotron (IC) resonance on the poloidal inhomogeneity of the density of the cyclotron-heated ion species. This additional effect has a significant impact on the poloidal density asymmetries due to radio-frequency ( $\mathrm{RF}$ ) heating, a key issue for high- $\mathrm{Z}$ impurity transport, leading, in particular, to a reduced ICRF (Ion-Cyclotron Radio-Frequency) heating impact on the in-out accumulation of high- $\mathrm{Z}$ impurities when the IC resonance is located on the high-field side.
\end{abstract}

\section{Introduction}

Plasma heating with radio-frequency (RF) waves in the ion-cyclotron (IC) range of frequencies has an impact on the asymmetry of impurity densities $[1,2,3]$. As a consequence of the ICRF heating, the density of the IC-resonant species becomes poloidally inhomogeneous. In compliance with the charge-neutrality constraint, plasma reacts to this inhomogeneity with an electrostatic potential which varies poloidally. For high-Z species this electrostatic potential is responsible of an in-out (on the inboard side) asymmetry of their density also in the presence of the centrifugal force in rotating plasmas. The possibility of influencing this electrostatic potential by ICRF heating was already investigated in [4] (and citations therein). In the frame of neoclassical theory in rotating axisymmetric plasmas [5], the effects of ICRF heating are taken into account by approximating the distribution of the heated species with a bi-Maxwellian characterized by $T_{\perp} / T_{\|}>1[6]$. This produces a set of consistent zeroth-order moment equations, which are strictly valid only on the high-field side (HFS) of the IC resonance position. This model, however, neglects an additional effect of the inhomogeneity of the confining magnetic field along the field lines (mirror effect) on the distribution 
function of the ICRF-heated ion species, as explained in the following. A peculiarity of cyclotron heating is to increase the perpendicular component of the velocity of the resonant ions $\left(v_{\perp}\right.$, where here parallel and perpendicular refer to the local direction of the confining magnetic field). Because of the mirror effect associated with the magnetic field inhomogeneity along the field lines, resonant particles become trapped when $v_{\perp} / v$ is increased beyond a certain threshold. Resonating trapped particles experience an increase of $v_{\perp}$ till when the tips of their banana orbit fall on the crossing points (hereafter tagged as resonance points) between the IC resonance surface (almost vertical) and the magnetic surface. Increasing further $v_{\perp}$ implies the exit of the ion from the IC resonance. This peculiar feature of cyclotron heating entails an increase of the flow of passing particles towards the trapped region in velocity space, and an accumulation of resonating particles in the proximity of the resonance points, feature experimentally confirmed in [7], for instance. In the previous work [6], the mirror effect on the motion of resonant species was considered without taking into account the additional effects due to the accumulation of resonating particles in the proximity of the resonance points. The impact of these additional effects on the in-out asymmetry of impurity density is precisely the object of the present study.

For clarity, in the following we reserve the name of bi-Maxwellian only to the product of two Gaussians depending separately on $v_{\perp}$ and on $v_{\|}$, and characterized by two different temperatures, $T_{\perp}$ and $T_{\|}$, respectively [9]. The bi-Maxwellian is the approximation for the distribution function of the ICRF-heated species assumed in [2, 6]. However, the bi-Maxwellian model must be modified to account for the toroidal mirror effect also on the wave-ion resonance, responsible for the "localization" of the IC resonance. In particular, on the LFS of the IC resonance the distribution functions of the resonating species develop the well-known "rabbits ears" [10] due to the accumulation of trapped ions turning just before the resonance itself (upper-left frame of figure 1). The biMaxwellian model of [6] ignores these "rabbits ears", and, therefore, accounts only for part of the density inhomogeneity induced by ICRF heating. To overcome this limitation, in the next Section we discuss an analytical model which accounts for these additional effects, originally proposed by Dendy and co-workers [8] to study the impact of ICRF on MHD stability (hereafter we call it Dendy's model to distinguish it from the pure bi-Maxwellian distribution function assumed in [6]), and afterwards used to investigate the impact of ICRF on equilibria $[11,12]$ and on the in-out asymmetry of the density of high-Z species [13]. Despite its unavoidable limitations, Dendy's model is useful to express in closed form the moments of the distribution function. In Section 3, the parallel momentum equation (9) of [6] is extended to account for the rabbit ears. Although this is achieved by renouncing a full consistency with the Fokker-Planck (FP) equation on the LFS of the IC resonance position, the simplified extension allows us to highlight the physical mechanisms responsible for the dependence of the poloidal density asymmetry on the position of the IC resonance, with significant consequences on the poloidal accumulation of high-Z impurity. A few examples of the dependence of the in-out asymmetry of high-Z impurity on the IC resonance position are given by using 
Dendy's model for the poloidal variation of the density of the ICRF-heated species in place of the numerical solution of the FP equation for the distribution function of the ICRF-heated species.

\section{Model of the distribution function of ICRF-heated species}

A first approximation of the distribution function of the ICRF-heated species, which accounts for an increase of the perpendicular kinetic energy due to ICRF, is the biMaxwellian with the perpendicular temperature larger than the parallel one, $T_{\perp}>$ $T_{\|}[14]$

$$
f_{\text {Maxw }}\left(v_{\perp}, v_{\|}\right)=\left(\frac{m}{2 \pi}\right)^{3 / 2} \frac{n}{T_{\perp} T_{\|}^{1 / 2}} \exp \left\{-\frac{m v_{\|}^{2}}{2 T_{\|}}\right\} \exp \left\{-\frac{m v_{\perp}^{2}}{2 T_{\perp}}\right\} .
$$

However, to describe the density accumulation around the resonance points it is necessary to go beyond the bi-Maxwellian. For this purpose, Dendy et al [8] proposed

$$
f_{\text {fast }}(\mu, \mathcal{E}, r)=\left(\frac{m}{2 \pi}\right)^{3 / 2} \frac{n_{\text {ic }}(r)}{T_{\perp, \text { ic }} T_{\|, \mathrm{ic}}^{1 / 2}} \exp \left\{-\frac{\mu B_{\text {ic }}}{T_{\perp, \text { ic }}}-\frac{\left|\mathcal{E}-\mu B_{\text {ic }}\right|}{T_{\|, \text {ic }}}\right\}
$$

where $\mu=m v_{\perp}^{2} / 2 B$ is the magnetic moment ( $B$ the module of the local confining magnetic field), $\mathcal{E}$ the particle energy, and $r$ the magnetic surface label. The subscript "ic" stands for the corresponding quantity evaluated a the IC resonance points on the magnetic surface labeled by $r$. In the limit of zero-banana width, Dendy's distribution function (2) is a function of the constants of motion. For simplicity, in the examples shown hereafter we consider the limit of circular tokamaks of large aspect ratio characterized by magnetic field of amplitude $B(r, \vartheta)=B_{0} R_{0} / R(r, \vartheta)$, where $B_{0}$ is its on-axis value, $R(r, \vartheta)=R_{0}(1+\epsilon(r) \cos \vartheta)$ with $R_{0}$ the major radius of the torus, $r$ and $\vartheta$ the radial and poloidal coordinates, and $\epsilon(r)=r / R_{0}$ the inverse-aspect ratio of the magnetic surface. However, the behaviour and trends discussed here are not limited by this approximation, since in all generality one can use the amplitude of $B$ as running coordinate along the magnetic field line. Figure 1 shows the contour plots of the distribution function of the minority ICRF-heated species according to (2) at different poloidal angles. At the point of minimum magnetic field, here the outer midplane point $(\vartheta=0$ - hereafter, values at this point are tagged with "lfs"), the distribution function has the typical bumps ("rabbit ears") of the ICRF-heated distribution function, located in the domain of the trapped particles. These rabbit ears are aligned along the boundary defined by those trapped particles with their banana tips on the IC resonances $\vartheta_{\text {ic }}[10,15]$. By using the constancy of $\mathcal{E}, \mu$ and $r$, the distribution function can be mapped along the magnetic field: the bumps coalesce along $v_{\|}=0$ at the poloidal position $\vartheta_{\text {ic }}$, and from the IC resonance points toward the HFS $f_{\text {fast }}$ is well described by a bi-Maxwellian with $\left(n_{\text {ic }}, T_{\perp, \text { ic }}, T_{\|, \text {ic }}\right)$.

The limited RF power per resonant particle and collisional isotropization make the 

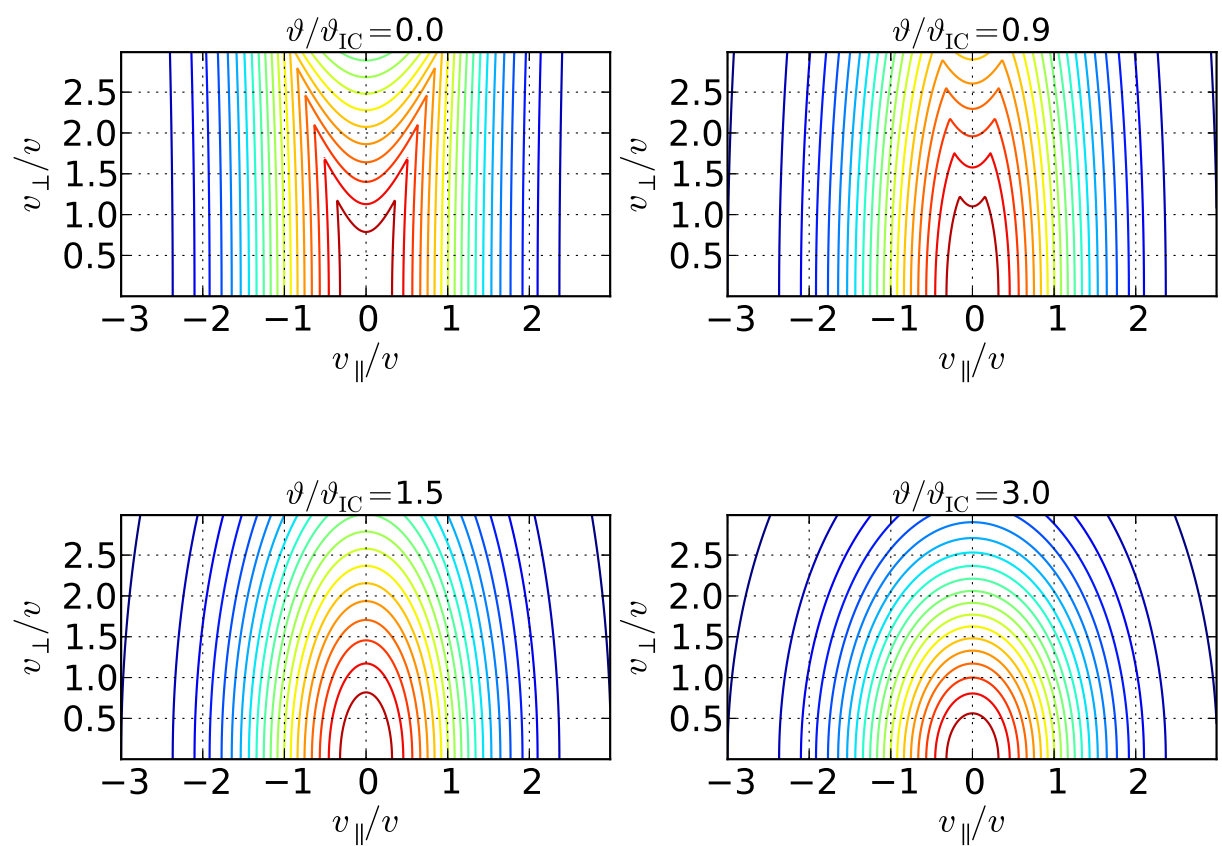

Figure 1. Distribution function for $\epsilon=0.1$ and $\vartheta_{\text {ic }}=\pi / 3$ according to Dendy's model (2).

ICRF heating accelerate to high energies only a finite fraction of the resonating species. Dendy's model (2) can describe only the fast anisotropic part of the distribution function of the ICRF-heated species. In fact, if $T_{\perp \text { ic }}=T_{\| \text {ic }}$ in $(2), f_{\text {fast }}$ becomes a Maxwellian only when the IC resonance is on the minimum of the magnetic field, $B_{\text {ic }}=B_{\mathrm{lfs}}$. Therefore, (2) cannot describe the thermal bulk, which is typically much closer to a Maxwellian. Thus, the whole distribution function can be described with a fast tail (2) superposed to a Maxwellian, $f_{\text {Maxw }}$, for the thermal background

$$
f(\mu, \mathcal{E}, r)=\left(1-\eta_{\text {fast }}\right) f_{\text {Maxw }}(\mathcal{E}, r)+\eta_{\text {fast }} f_{\text {fast }}(\mu, \mathcal{E}, r),
$$

with $\eta_{\text {fast }}$ the fraction of fast IC-accelerated ions, which can be considered as a fitting parameter as well as $T_{\| \text {,ic }}$ and $T_{\perp, \text { ic }}$.

In the case of IC minority heating the RF power per resonating ion is typically high enough to cause a substantial deformation of the distribution function of the minority. Therefore, for common operational parameters, describing the whole minority distribution function with (2) is an acceptable approximation for the minority ICRFheated species, and we use it in the following analysis (it corresponds to (3) with $\eta_{\text {fast }}=1$ ). This approximation is not always so good for the IC heating of the majority species at harmonics of the IC frequency.

The approximation (2) makes possible to express density and temperatures in closed forms as function of $\mathcal{T}=T_{\perp, \text { ic }} / T_{\| \text {,ic }}$ and $\mathcal{B}=B_{\text {ic }} / B$ (where $\mathcal{B}<1$ and $\mathcal{B}>1$ imply to be on the HFS and LFS of the IC resonance, respectively) [16]. The density along the magnetic field line is

$$
n(r, \mathcal{B} ; \mathcal{T})=n_{\text {ic }}(r ; \mathcal{T}) \mathcal{N}(r, \mathcal{B} ; \mathcal{T})
$$




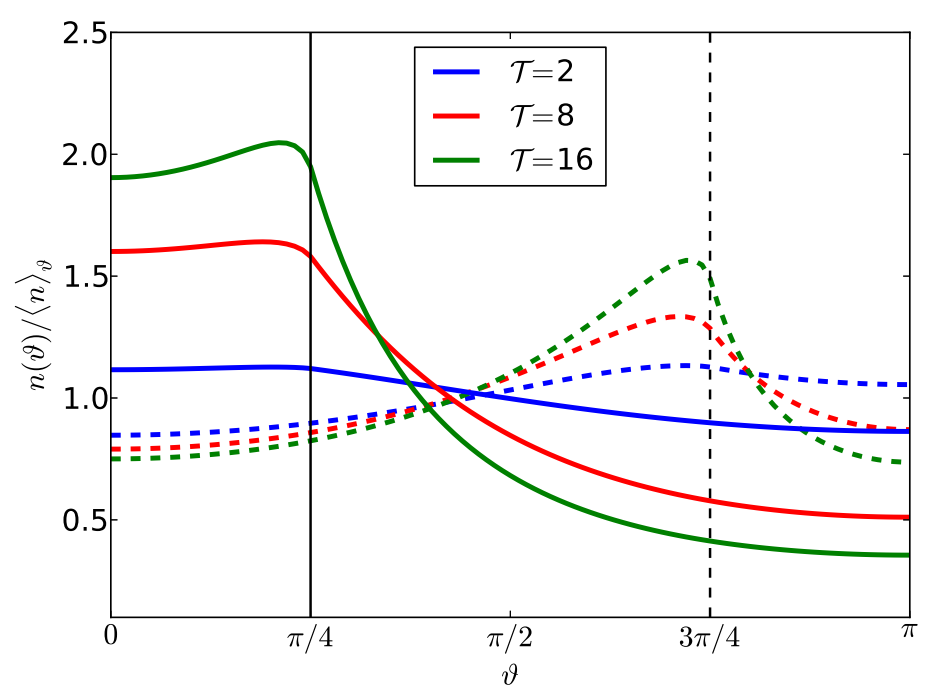

Figure 2. Poloidal variation of the density of the ICRF-heated ion species when mapping the distribution function from the low-field side (LFS) $\vartheta=0$ to the high-field side (HFS) $\vartheta=\pi$. The IC resonance position (vertical lines) is at $\pi / 4$ (LFS) for solid lines and at $3 \pi / 4$ (HFS) for dashed lines.

with the density form factor, $\mathcal{N}$,

$$
\mathcal{N}(r, \mathcal{B} ; \mathcal{T})=\mathscr{T}_{-}\left[1+\left(\frac{\mathscr{T}_{+}}{\mathscr{T}_{-}}-1\right) \sqrt{\frac{\mathcal{T}}{\mathcal{B}}(\mathcal{B}-1)} H(\mathcal{B}-1)\right]
$$

the coefficients $\mathscr{T}_{ \pm}=[\mathcal{B} \pm(\mathcal{B}-1) \mathcal{T}]^{-1}$, and $H(x)$ is the Heaviside function. The density at the IC resonance points (i.e. at $\mathcal{B}=1$ ) is defined with respect to its surface-averaged value, namely $n_{\text {ic }}(r ; \mathcal{T})=\langle n\rangle /\langle\mathcal{N}\rangle$, where the angular brackets denote the surface average. Figure 2 shows the poloidal modulation of the density when moving from the low-field side (LFS, $\vartheta=0, \mathcal{B}=\mathcal{B}_{\text {max }}$ ) to the high-field side $\left(\mathrm{HFS}, \vartheta=\pi, \mathcal{B}=\mathcal{B}_{\min }\right.$ ) through the resonance points $\vartheta_{\text {ic }}$ (vertical lines). A density maximum is present around the IC resonance points, slightly shifted on the LFS. The origin of this maximum is easily understood from the definition of the density as zero-order moment of $f$ in $(\mu, \mathcal{E})$ coordinates

$$
n(r ; B)=4 \pi \int_{0}^{+\infty} \mathrm{d} \mu \int_{\mu B}^{+\infty} \mathrm{d} \mathcal{E} \frac{B}{v_{\|}} f(\mu, \mathcal{E}, r),
$$

where $v_{\|}=\sqrt{2(\mathcal{E}-\mu B)}$, valid in the absence of an electrostatic potential. The dependence on $B$ is equivalent to the dependence on the poloidal angle $\vartheta$. The $v_{\|}^{-1}$ factor in the Jacobian $\left(J \equiv B / v_{\|}\right)$weights more those particles that have one of their turning points at $\vartheta$, since they stay longer there having $v_{\|} \rightarrow 0$. Thus, the density experiences a maximum when the bumps coalesce at the $v_{\|}=0$ line. In passing, we observe that the Maxwellian is invariant with respect to the $\vartheta$ mapping, and thus the density is constant in $\vartheta$.

As shown in figure 2, when the IC resonance is moved from the LFS (solid lines) to the HFS (dashed lines), the density peak moves towards the HFS, inverting the effects of 
the density inhomogeneity of the ICRF-heated minority on the high-Z impurities, and precisely acting in favour of the centrifugal force. Additionally, the poloidal variation of the density with increasing $\mathcal{T}$ is less pronounced when the IC resonance is shifted from the LFS to the HFS.

The parallel and perpendicular energies defined as second order moments of (2) are $[16]$

$$
\begin{aligned}
& \frac{W_{\|}(r, \mathcal{B} ; \mathcal{T})}{n_{\mathrm{ic}}(r ; \mathcal{T}) T_{\|, \mathrm{ic}}}=\frac{\mathscr{T}_{-}}{2}\left\{1+\mathrm{H}(\mathcal{B}-1)\left[\mathcal{T}\left(1-\mathcal{B}^{-1}\right)\right]^{3 / 2}\left(\frac{\mathscr{T}_{+}}{\mathscr{T}_{-}}-1\right)\right\}, \\
& \frac{W_{\perp}(r, \mathcal{B} ; \mathcal{T})}{n_{\mathrm{ic}}(r ; \mathcal{T}) T_{\perp, \mathrm{ic}}}=\mathscr{T}_{-}^{2}+H(\mathcal{B}-1) \sqrt{\mathcal{T}\left(1-\mathcal{B}^{-1}\right)}\left[\frac{\mathscr{T}_{+}-\mathscr{T}_{-}}{2 \mathcal{B}}+\left(\mathscr{T}_{+}^{2}-\mathscr{T}_{-}^{2}\right)\right],
\end{aligned}
$$

The bi-Maxwellian distribution function with the same energy content as Dendy's

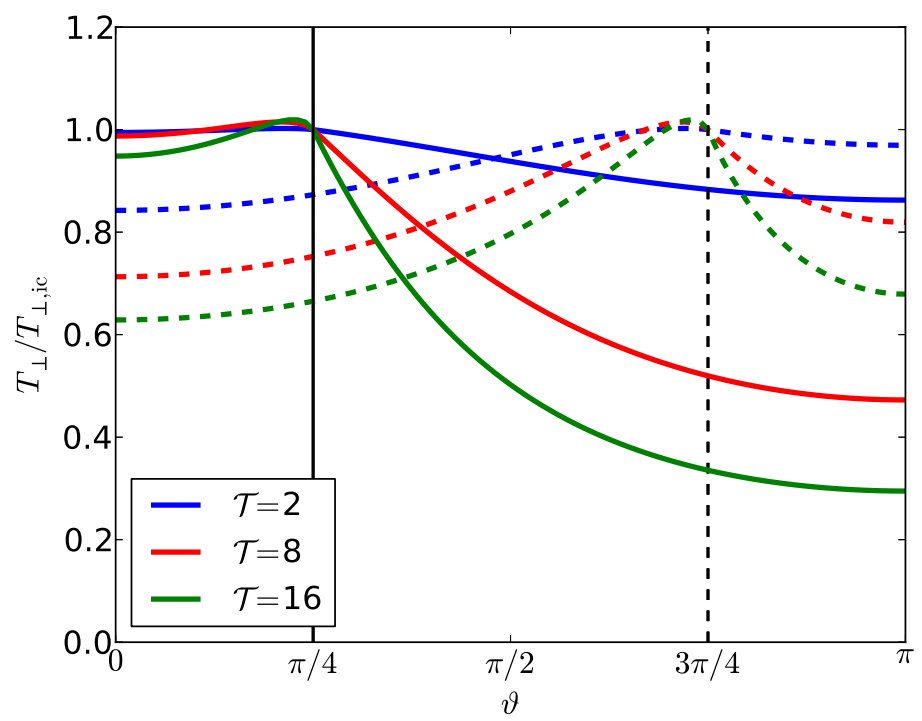

Figure 3. Poloidal variation of $T_{\perp}$ of the ICRF-heated ion species for the same parameters as in figure 2 .

function has the effective parallel and perpendicular temperatures defined by $W_{\|}$and $W_{\perp}$

$$
\frac{T_{\|}}{T_{\|, \mathrm{ic}}}=2 \frac{W_{\|}(r, \mathcal{B} ; \mathcal{T})}{n(r, \mathcal{B} ; \mathcal{T}) T_{\|, \mathrm{ic}}}, \quad \frac{T_{\perp}}{T_{\perp, \mathrm{ic}}}=\frac{W_{\perp}(r, \mathcal{B} ; \mathcal{T})}{n(r, \mathcal{B} ; \mathcal{T}) T_{\perp, \mathrm{ic}}} .
$$

Figures 3 and 4 show the poloidal dependence of $T_{\perp}$ and $T_{\|}$, respectively, for the same values of $\mathcal{T}$ as in figure 2. On the high field side of the IC resonance, $T_{\|}$is constant and $T_{\perp} / T_{\perp, \text { ic }}=\mathscr{T}_{-}$, as in the case of the bi-Maxwellian [6]. The poloidal variations of $T_{\perp}$ and $T_{\|}$decrease when the IC resonance is shifted towards the HFS. For completeness the dependence of $T_{\perp} / T_{\|}$on the poloidal angle is shown in figure 5 . The value of $T_{\perp} / T_{\|}$ on the outer midplane point decreases substantially when moving the IC resonance from the LFS to the HFS. Simulations predict values of $\mathcal{T}$ that can be slightly larger than 


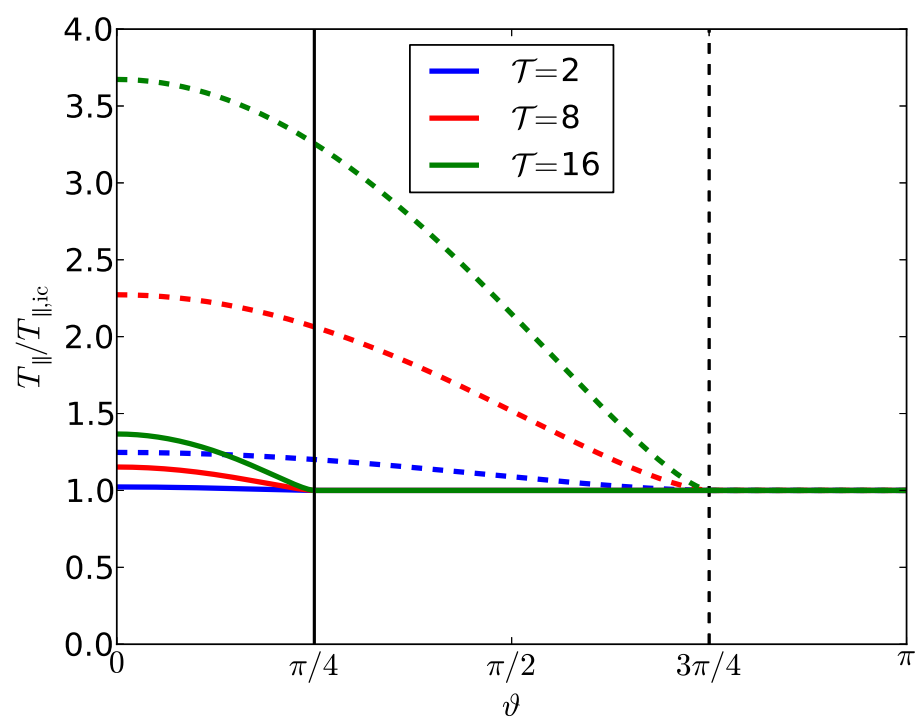

Figure 4. Poloidal variation of $T_{\|}$of the ICRF-heated ion species for the same parameters as in figure 2. On the high-field side of the IC resonance $T_{\|}$is constant.

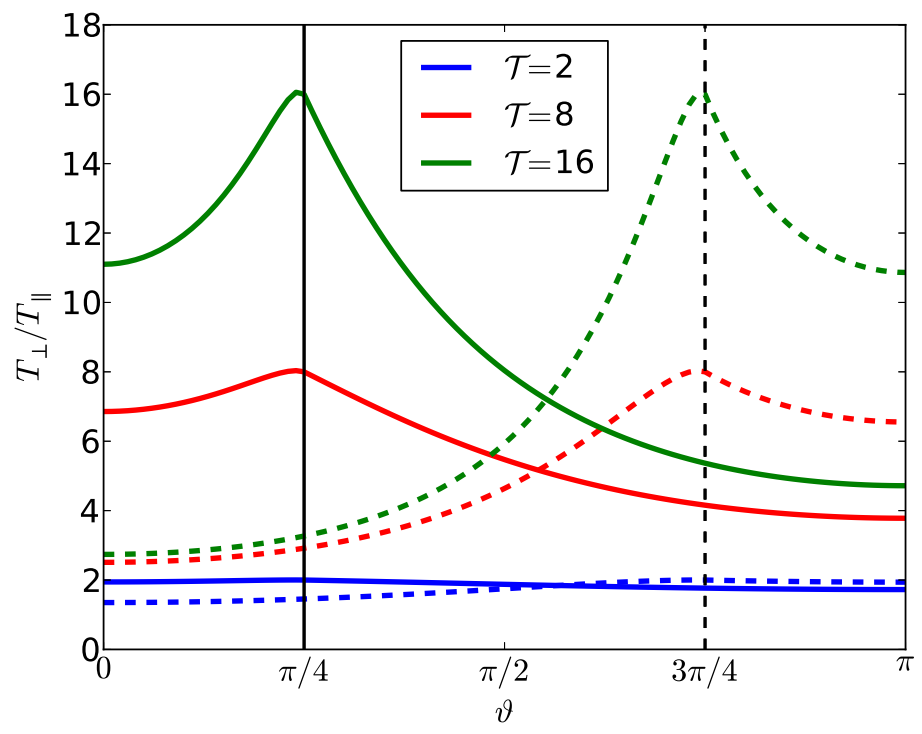

Figure 5. Poloidal variation of $T_{\perp} / T_{\|}$of the ICRF-heated ion species for the same parameters as in figure 2 .

10 for the present medium-sized plasmas, and lower for large devices, such as JET [3], mainly as a consequence of the lower RF power per resonant particle.

\section{Model equations for the density asymmetry induced by ICRF-heated species}

The density inhomogeneity along the magnetic field of the ICRF-heated ions species (see figure 2) entails an electrostatic potential that acts on ions and electrons [2]. Especially 
for high-Z ions, such as tungsten and molybdenum, the resulting electrostatic force can even invert the density out-in (on the outboard side) asymmetry due to the centrifugal force in rotating plasmas.

If the distribution of the ICRF-heated species is approximated with (2), the parallel momentum equation must have (4) as solution in the absence of centrifugal forces and electrostatic potentials. Renouncing a consistent derivation, as done for the biMaxwellian distribution functions [6], a set of approximate equations modeling the impact on impurity asymmetries is (see appendix)

$$
\begin{aligned}
& \frac{\nabla_{\|} n_{j}}{n_{j}}=-Z_{j} \frac{\nabla_{\|}(e \tilde{\Phi})}{T_{j, \|}}+\frac{\nabla_{\|} \mathcal{N}_{j}}{\mathcal{N}_{j}}+\frac{m_{j}}{2} \frac{\nabla_{\|} V_{j}^{2}}{T_{j, \|}}, \\
& n_{e}=\sum_{j} Z_{j} n_{j}, \\
& \frac{e \tilde{\Phi}}{T_{e}}=\ln \frac{n_{e}}{n_{e, l \mathrm{lfs}}}, \\
& \mathcal{N}(r, \mathcal{B} ; \mathcal{T})=\mathscr{T}_{-}\left[1+\left(\frac{\mathscr{T}_{+}}{\mathscr{T}_{-}}-1\right) \sqrt{\frac{\mathcal{T}}{\mathcal{B}}(\mathcal{B}-1)} H(\mathcal{B}-1) H(\mathcal{T}-1)\right],
\end{aligned}
$$

where $\mathscr{T}_{ \pm}=[\mathcal{B} \pm(\mathcal{B}-1) \mathcal{T}]^{-1}$ and $V=\omega_{\varphi}(r) R$ is the plasma toroidal rotation with $\omega_{\varphi}$ the corresponding angular frequency. The third term on the RHS of the first equation is the centrifugal force. The electron force balance equation (third equation) couples the electrostatic potential $\tilde{\Phi}$ with the electron density, $n_{e}$, which is constrained by the charge neutrality (second equation). The main difference of the first of (9) with equation (9) of [6] is the second term on the LHS, which now depends on the density form factor $\mathcal{N}$. The effects of ICRF heating enter via $\mathcal{N}$ of the resonant species. On the HFS of the IC resonance these equations are exactly equations (13) of [6] with the first replaced by equation (9) of [6]. The additional Heaviside function dependent on $\mathcal{T}$ in the expression for $\mathcal{N}$ guarantees that for those plasma species not resonating with the waves $\left(\mathscr{T}_{-}=\mathscr{T}_{+}=\mathcal{T}=1\right)$, the second term on the right-hand side of the parallel momentum equation is equal to zero. The set of equations (9) has been solved as an initial value problem with the concentrations $n_{j} / n_{e}$ given on the outer midplane point. To keep fixed the average concentration of the (minority) ICRF-heated species, a recursive loop based on the secant method has been employed to solve (9) [6]. Alternatively, the first equation (9) can be integrated in closed form,

$$
\frac{n_{j}}{n_{j, \mathrm{lfs}}}=\frac{\mathcal{N}_{j}}{\mathcal{N}_{j, \mathrm{lfs}}} \exp \left\{Z_{j} \frac{e \tilde{\Phi}}{T_{j, \|}}+A_{j} M_{\varphi}^{2} \frac{T_{i}}{T_{j, \|}}\left[\left(\frac{R}{R_{\mathrm{lfs}}}\right)^{2}-1\right]\right\}
$$

with $A_{j}=m_{j} / m_{p}, m_{p}$ the proton mass, and $M_{\varphi}=\omega_{\varphi} R_{\mathrm{lfs}} / \sqrt{2 T_{i} / m_{p}}$ the Mach number of protons at the background ion temperature, $T_{i}$, and on the reference LFS position, $R_{\mathrm{lfs}}$. When the first equation of (9) is replaced with (10), (9) becomes a set of algebraic equations, whose solution can be found with a root finder. 
As done in $[17,6]$, it is worthwhile deriving an approximate solution for the density of the high-Z impurities in closed form. We start by approximating all the ion species with an effective species characterized by the charge, $Z_{\mathrm{eff}}=\sum_{i} Z_{i}^{2} n_{i} / n_{e}$ and the mass $m_{\mathrm{eff}}=m_{p} A_{\mathrm{eff}}=\sum_{j} m_{j} Z_{j} n_{j} / n_{e}$, and with the density given by the charge neutrality $\tilde{n}_{i}=n_{e} / Z_{\text {eff }}$. If the plasma rotates toroidally, $\tilde{n}_{i}$ is given by (10). By using the electron force balance $n_{e} / n_{e, \mathrm{lfs}}=\exp \left\{e \tilde{\Phi} / T_{e}\right\}$ (in the case of electrons the centrifugal force is neglected because of the smallness of their mass) and charge neutrality, the electrostatic potential is linked to the plasma rotation by

$$
\frac{e \tilde{\Phi}}{T_{e}}=\frac{T_{i}}{T_{i}+Z_{\mathrm{eff}} T_{e}} A_{\mathrm{eff}} M_{\varphi}^{2}\left[\left(\frac{R}{R_{\mathrm{lfs}}}\right)^{2}-1\right]
$$

A minority ICRF-heated species perturbs the electrostatic potential $\tilde{\Phi}, \tilde{\Phi}=\tilde{\Phi}^{(0)}+\delta \tilde{\Phi}$, with $\tilde{\Phi}^{(0)}$ given by equation (11). Since in typical ICRF minority scenarios the concentration $n_{m}$ of the minority resonating species $\left(m_{m}, Z_{m}\right)$ is much smaller than the concentration of the majority species, $n_{m} Z_{m} \ll n_{M} Z_{M}$, $\delta \tilde{\Phi}$ can be determined pertubatively by keeping only the first-order terms in the Taylor expansions. The perturbation $\delta \tilde{\Phi}$ is obtained by equating the expression of $\delta n_{e}$ from the charge neutrality, $\delta n_{e}=Z_{m} n_{m}+Z_{\text {eff }} \delta \tilde{n}_{i}$, with $\delta n_{e}$ from the electron balance force equation, $\delta n_{e} / n_{e, l f s}=$ $\left(e \delta \tilde{\Phi} / T_{e}\right) \exp \left\{e \tilde{\Phi}^{(0)} / T_{e}\right\}$. To determine $\delta \tilde{n}_{i}$, we observe that

$$
Z_{\text {eff }} \tilde{n}_{i}=\left(n_{e, \mathrm{lfs}}-Z_{m} n_{m, \mathrm{lfs}}\right) \exp \left\{-Z_{\mathrm{eff}} \frac{e \tilde{\Phi}}{T_{i}}+A_{\mathrm{eff}} M_{\varphi}^{2}\left[\left(\frac{R}{R_{\mathrm{lfs}}}\right)^{2}-1\right]\right\}
$$

which expanded to the first-order gives

$$
Z_{\text {eff }} \delta \tilde{n}_{i}=-\left(Z_{\text {eff }} n_{e, \mathrm{lfs}} \frac{e \delta \tilde{\Phi}}{T_{i}}+Z_{m} n_{m, \mathrm{lfs}}\right) \exp \left\{\frac{e \tilde{\Phi}^{(0)}}{T_{e}}\right\} .
$$

If $A_{m}$ and $Z_{m}$ of the ICRF-heated species are not too different from $A_{\text {eff }}$ and $Z_{\text {eff }}$, the density of the ICRF-heated species can be approximated with $n_{m} / n_{m, \text { lfs }}=$ $\mathcal{N}_{m} / \mathcal{N}_{m, \text { lfs }} \exp \left\{e \tilde{\Phi}^{(0)} / T_{e}\right\}$, and $\delta \tilde{\Phi}$ is

$$
\frac{e \delta \tilde{\Phi}}{T_{e}}=\frac{Z_{m} T_{i}}{T_{i}+Z_{\mathrm{eff}} T_{e}} \frac{n_{m, \mathrm{lfs}}}{n_{e, \mathrm{lfs}}}\left[\frac{\mathcal{N}_{m}}{\mathcal{N}_{m, \mathrm{lfs}}}-1\right] .
$$

To the first-order in the perturbation, an approximate expression of the density of impurities in a rotating plasma heated with ICRF waves becomes

$$
\begin{aligned}
\ln \left(\frac{n_{Z}}{n_{Z, \mathrm{lfs}}}\right)=- & Z_{Z} \frac{Z_{m} T_{e}}{T_{i}+Z_{\mathrm{eff}} T_{e}} \frac{n_{m, \mathrm{lfs}}}{n_{e, \mathrm{lfs}}}\left[\frac{\mathcal{N}_{m}}{\mathcal{N}_{m, \mathrm{lfs}}}-1\right]+ \\
& {\left[1-\frac{m_{\mathrm{eff}}}{m_{Z}} \frac{Z_{Z} T_{e}}{T_{i}+Z_{\mathrm{eff}} T_{e}}\right] A_{Z} M_{\varphi}^{2}\left[\left(\frac{R}{R_{\mathrm{lfs}}}\right)^{2}-1\right] . }
\end{aligned}
$$

This is the heuristic expression for the impurity density we were looking for, where $\mathcal{N}_{m} / \mathcal{N}_{m \text {,lfs }}$ can be estimated either with Dendy's model (5) or from the zeroth-order moment of the numerical solution of the FP equation. 


\section{Discussion}

As typical ICRF scenario [2, 3], we consider ICRF heating of minority hydrogen $(\mathrm{H})$ in deuterium (D) plasmas, and its impact on the in-out asymmetry of tungsten (W). For $\mathcal{B}<1$ (i.e. on the HFS with respect to the IC resonance position), (2) becomes a bi-Maxwellian with $T_{\|}$constant and $T_{\perp} / T_{\perp, \text { ic }}=\mathscr{T}_{-}$, and equations (9) become exactly those of [6]. At a given magnetic surface, for various values of $\mathcal{T}_{H}=T_{\perp, H} / T_{\|, H}$ at the
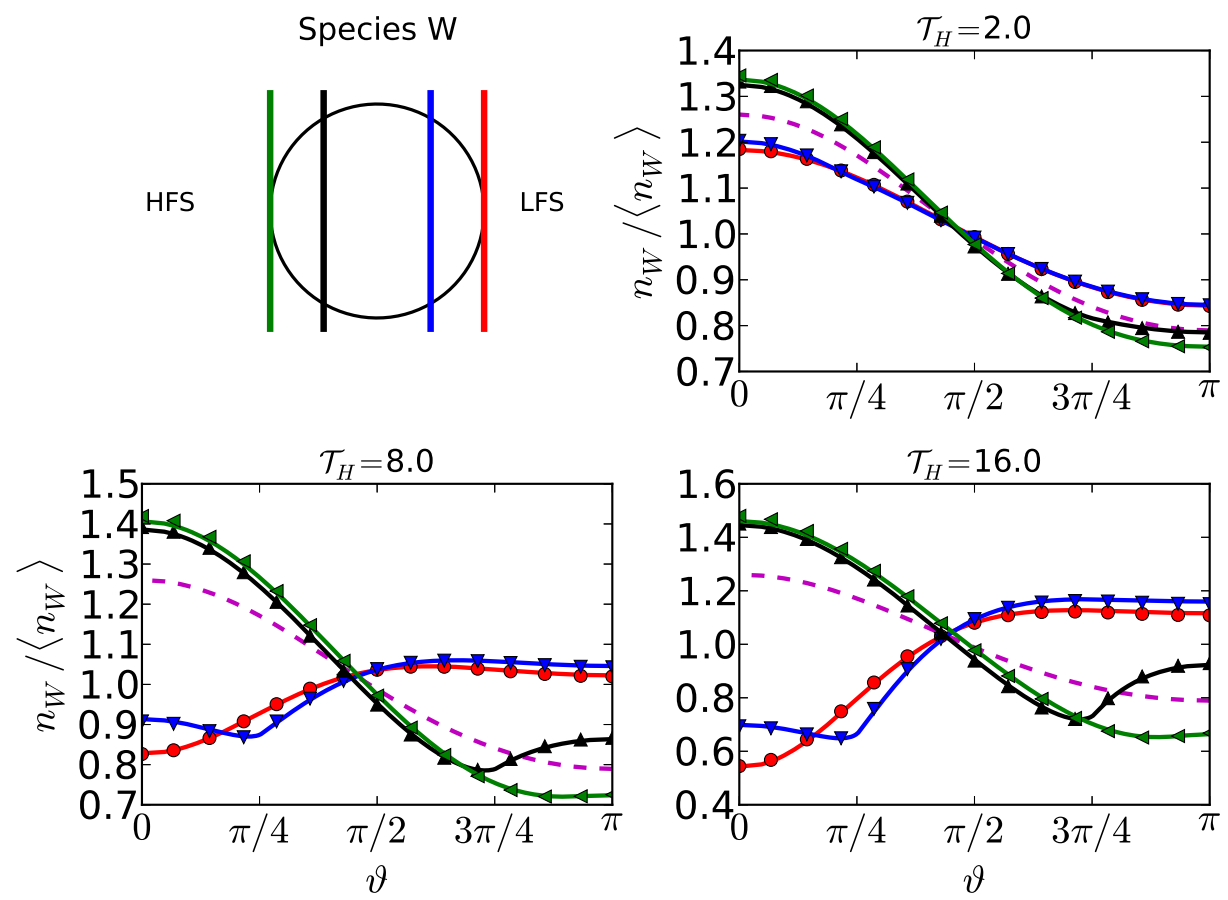

Figure 6. Poloidal variation of the tungsten concentration on a given magnetic surface as function of $R / R_{\mathrm{lfs}}=(1+\epsilon \cos \vartheta) /(1+\epsilon)$ with $\epsilon=0.1, T_{e}=T_{\|}=2 \mathrm{keV}$ for an ionization state of $\mathrm{W}$ equal to 33 . The ICRF-heated hydrogen concentration is $5 \%$ and the proton Mach number is 0.1. The solid lines are solution of (9), whereas the symbols are obtained with the approximated formula (15). The colors refer to the resonance position, as sketched in the first frame. The LFS and HFS are respectively at $\vartheta=0$ and $\vartheta=\pi$. In the case of the red lines the distribution function of the ICRF-heated species is a bi-Maxwellian, as in the case of [6]. The dashed-magenta line shows the poloidal density of $\mathrm{W}$ in the absence of the effects of the ICRF-heated minority.

IC resonance and different positions of the IC resonance, figure 6 shows the poloidal variations of the tungsten density in the case of deuterium plasmas rotating at the toroidal (proton) Mach number $M_{\varphi}:=\omega_{\varphi} R_{\mathrm{lfs}} / \sqrt{2 T_{\|} / m_{p}}=0.1$, with $5 \%$ of ICRFheated hydrogen. For comparison, the magenta dashed line is calculated in the absence of temperature anisotropies, and thus only in the presence of the centrifugal effect. The colors refer to the resonance position, as sketched in the first frame of figure 6 . The solid lines are solution of (9), whereas the symbols are obtained with the heuristic formula (15). The agreement between the numerical solution of (9) and the profile (15) is very good, and the lines overlap almost everywhere. In the case of tangent resonances 
on the LFS (red lines), the distribution function of the minority is exactly the biMaxwellian of the case studied in [6]. When $\mathcal{T}_{H}$ increases the $\mathrm{W}$ density develops a

Species H

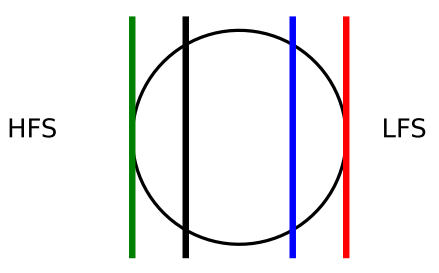

$\mathcal{T}_{H}=8.0$

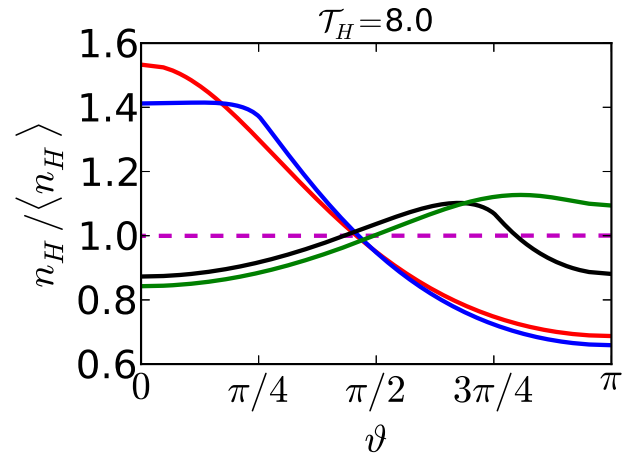

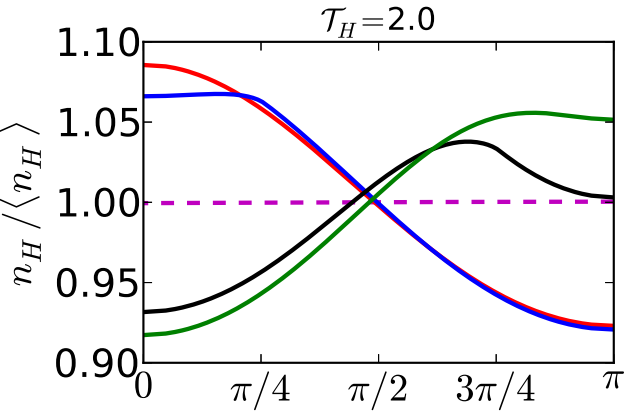

$\mathcal{T}_{H}=16.0$

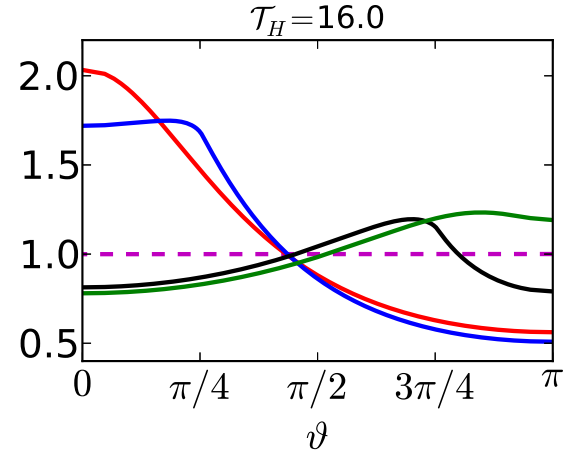

Figure 7. Akin to figure 6 for the minority ICRF-heated species.

minimum close to the IC resonance, and precisely on the LFS of the IC resonance. This minimum corresponds to the maximum of the poloidal density profiles of the ICRFheated minority species, as shown in figure 7. Additionally, the amplitude of the density variation of the ICRF-heated species increases with $\mathcal{T}_{H}$, and this increase is larger when the IC resonance is on the LFS. The impact of ICRF heating on the in-out asymmetry of tungsten is shown in figure 8.a as function of $\mathcal{T}_{H}$ for two values of $M_{\varphi}$ and for the same IC resonance positions of figures 6 and 7 . The horizontal magenta lines are the reference value of the in-out asymmetry in the absence of ICRF heating. The amplitude and direction effect of ICRF heating depends substantially on the position of the IC resonance. When the IC resonance is on the HFS (green and black lines), depending on the values of $M_{\varphi}, \mathcal{T}_{H}$ and $\varepsilon$, the potential due to the density asymmetry of the ICRFheated species can even enhance the out-in asymmetry of the centrifugal force [2]. This example shows the general trend that the effect of minority-ICRF heating on the in-out asymmetry of high-Z impurities depends on the position of the IC resonance: This effect is larger and acts against the centrifugal effects when the IC resonance is on the LFS.

It is worthwhile comparing the present results with those obtained in [6] assuming bi-Maxwellian distribution functions. Figure 8.b shows the result for the bi-Maxwellian model with the values of the temperature anisotropy $\left.\mathcal{T}_{H, \mathrm{lfs}}=T_{\perp} / T_{\|}\right)_{\mathrm{lfs}}$ on the external midplane point (namely the values at $\vartheta=0$ in figure 5). This approach should be close to the analysis done in [2], where $\mathcal{T}_{H \text {,lfs }}$ is calculated with the FP solver embedded 

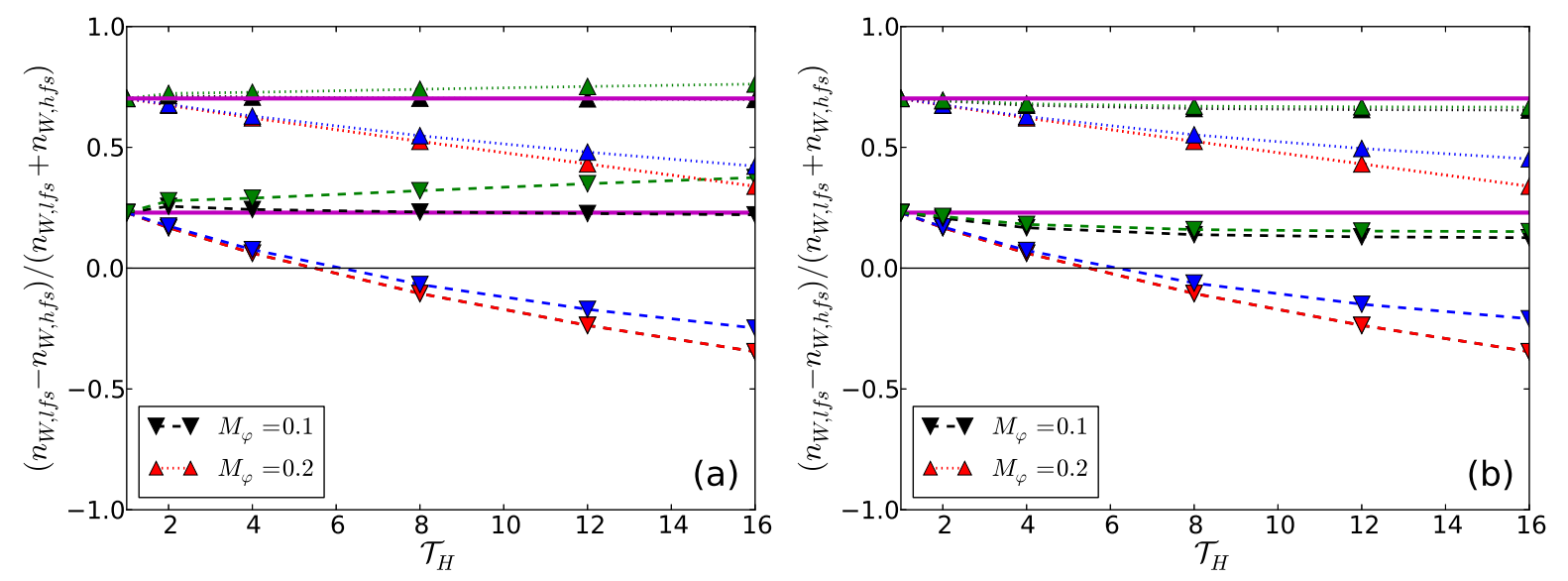

Figure 8. In-out asymmetry of $\mathrm{W}^{33+}$ as function of $\mathcal{T}_{H}$ for two values of the hydrogen Mach number. The colors refer to the IC resonance position according to figure 6 . The magenta horizontal lines are in correspondence of the in-out asymmetry in the presence of the only centrifugal effects. The plot (b) is for the bi-Maxwellian model [6] with $\mathcal{T}_{H}$ in equations (9) equal to the values of $T_{\perp} / T_{\|}$at $\vartheta=0$ of figure 5 .
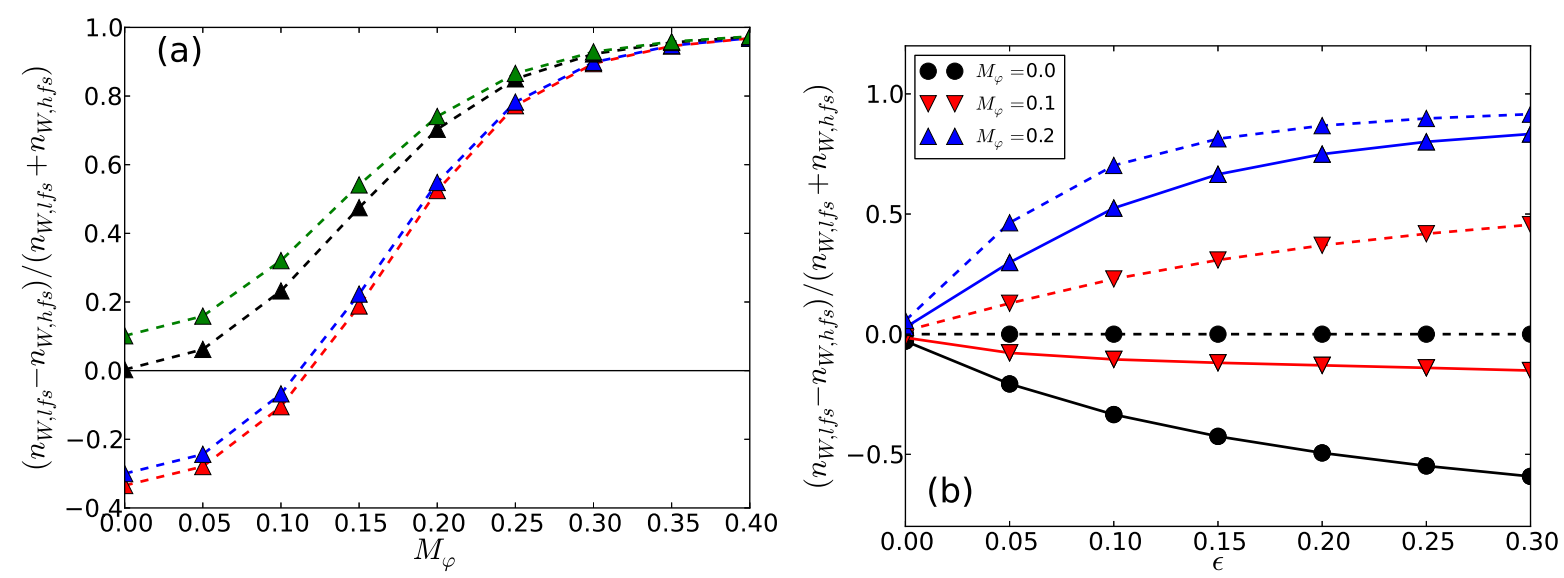

Figure 9. (a) In-out asymmetry of $\mathrm{W}^{33+}$ as function of the hydrogen Mach number $M_{\varphi}$ for $\mathcal{T}_{H}=8$ and $\epsilon=0.1$ (the inverse-aspect ratio of the magnetic surface). The colours have the same meaning of figures $6-8$.

(b) In-out asymmetry of $\mathrm{W}^{33+}$ as function of the inverse-aspect ratio, $\epsilon$, of the magnetic surface tangent to the IC resonance layer and for $\mathcal{T}_{H}=8$. In (b) the dashed lines show the corresponding in-out asymmetry in the absence of ICRF effects.

in TRANSP that solves the bounce-averaged FP equation at $R_{\mathrm{lfs}}$ and accounts for the trapping effects [18]. Therefore, part of the trapping effects are taken into account by the fact that $\mathcal{T}_{H \text {,lfs }}<\mathcal{T}_{H} \equiv \mathcal{T}_{H \text {,ic }}$. Figure 8.b shows the in-out asymmetry according to these additional approximations. Notice that in figures 8.a and 8.b the red lines are the same, and only the others are affected by the resonance localization. In particular, in figure 8.b all the lines are decreasing with $\mathcal{T}_{H}$ and the effect of ICRF heating is substantially reduced when the IC resonance is not tangent to the magnetic field on the LFS. In figure 9.a the in-out asymmetry of tungsten is shown as function of the 
hydrogen Mach number for $\mathcal{T}_{H}=8$. The range of $M_{\varphi}$ in figure 9.a largely covers the possible experimental values in the present devices $[19,20,21,22]$. The limit $M_{\varphi}=0$ corresponds to the case analyzed in [13].

In figure 9.b the in-out asymmetry, evaluated on the magnetic surface tangent to the IC resonance on the LFS, is shown as function of the inverse-aspect ratio, $\epsilon$. The solid blue and red lines refer to two different values of the hydrogen Mach number, whereas, for comparison, the black line shows the in-out asymmetry in the absence of the centrifugal

force. The dashed colored lines are the equivalent cases of the solid lines in the absence of ICRF effects. The ICRF effects on the in-out asymmetry increase with $\epsilon$. However, it is important to observe that the anisotropy parameter $\mathcal{T}$ depends mainly on the ICRF absorbed power per resonant particle and on the plasma collisionality. For the same amount of ICRF absorbed power, $\mathcal{T}$ decreases with the plasma volume and thus with $\epsilon$ of the magnetic surface tangent to the IC resonance. As a consequence, the increase of the in-out asymmetry with $\epsilon$ in figure $9 . \mathrm{b}$ is partially compensated by the decrease with $\epsilon$ of the temperature anisotropy of the ICRF-heated species.

\section{Conclusions}

ICRF heating makes poloidally inhomogeneous the density of the heated species. As back-reaction, an electrostatic potential appears in compliance with the charge neutrality. As a consequence of this electrostatic potential, weak in-out (on the inboard side) asymmetry of the density of high-Z impurities can appear also in the presence of centrifugal force, which acts instead for an accumulation on the outboard side (outin) $[1,2]$. This has beneficial effects on the impurity behaviour [23, 3]. With reference to the model discussed in [6], here we have added the effects of the spatial localization of the IC resonance responsible for the accumulation of resonating particle around the IC resonance, recognizable as "rabbit ears" in the distribution function of the ICRFheated species. As main result of this analysis we observe that, when the IC resonance is shifted from the LFS to the HFS, for the same value of the temperature anisotropy, $\mathcal{T}=T_{\perp, \text { ic }} / T_{\| \text {,ic }}$, the in-out impurity accumulation induced by ICRF heating does not only decrease in amplitude but it even changes sign becoming an out-in asymmetry which adds to the contribution of the centrifugal force. This behaviour can be only partially captured by the bi-Maxwellian model also in the case that $\mathcal{T}$ is reduced when shifting the IC resonance from the LFS to the HFS. For instance, this reduction of $\mathcal{T}$ is accounted for when it is calculated as the ratio of the corresponding second-order moments of the solution of the bounce-averaged Fokker-Planck (FP) equation, with the latter including trapping effects [2]. At any rate, the bi-Maxwellian model cannot

fully capture the amplitude reduction and foresee the change of sign of the impurity accumulation when the IC resonance is shifted on the HFS. Here, we have shown that a preciser modeling can be achieved by using the first three equations of (9) with the density form factor $\mathcal{N}$ of the ICRF-heated species calculated either directly from the numerical solution of the FP equation or from the Dendy's model (last equation in (9)) 
with $\mathcal{T}$ estimated from the second-order moments of the distribution function at the IC resonance position. Finally, to facilitate fast data analysis we have derived (15) as an approximated solution of (9), and we have shown that the agreement is very good.

\section{Acknowledgments}

We are grateful to J. P. Graves and M. L. Reinke for fruitful discussions, and to O. Maj for the use of his solver of algebraic-differential systems.

\section{Appendix A. Parallel momentum equation}

The parallel momentum equation (the first one in (9)) follows from the constraint that the coefficient of the term proportional to $v_{\|}$in the Fokker-Planck equation, written in the reference frame moving with the plasma velocity $\mathbf{V}$ (see equation (44) in [5] or (1) in [6]), vanishes

$$
\hat{b} \cdot\left\{\nabla f-\left[\frac{q}{m} \nabla \Phi+\mathbf{V} \cdot \nabla \mathbf{V}\right] \frac{1}{v_{\|}} \frac{\partial f}{\partial v_{\|}}\right\}=0,
$$

where $f$ is the distribution function, $\hat{b}=\mathbf{B} / B$ and $\mathbf{B}$ is the confining magnetic field. The distribution function $f$ is split as

$$
f\left(v_{\|}, v_{\perp}, \vartheta, \psi\right)=\tilde{n}(\vartheta, \psi) F\left(v_{\|}, v_{\perp}, \psi, \vartheta\right)
$$

where $F$ accounts for the impact of the ICRF heating and $\tilde{n}$ for the poloidal dependence of the density caused by the centrifugal force and the potential $\Phi$. With this choice the zeroth-order moment of $F$ is the form-factor of the density variation induced by the ICRF-driven pressure anisotropy, $\mathcal{N}$, defined in (5) for the case of Dendy's function. Thus, the total density of the ion species is $n=\tilde{n} \mathcal{N}$.

By defining the parallel temperature associated with $F$ as

$$
\frac{n}{T_{\|}}=-\frac{1}{m} \int \mathrm{d}^{3} v \frac{1}{v_{\|}} \frac{\partial f}{\partial v_{\|}},
$$

in close analogy with the definition of $T_{\|}$in the case of a bi-Maxwellian, and by observing that

$$
\frac{\nabla n}{n}=\frac{\nabla \tilde{n}}{\tilde{n}}+\frac{\nabla \mathcal{N}}{\mathcal{N}}
$$

the parallel momentum equation becomes

$$
\frac{\nabla_{\|} n}{n}=\frac{\nabla_{\|} \tilde{n}}{\tilde{n}}+\frac{\nabla_{\|} \mathcal{N}}{\mathcal{N}}=-q \frac{\nabla_{\|} \Phi}{T_{\|}}+\frac{\nabla_{\|}\left(m V^{2}\right)}{2 T_{\|}}+\frac{\nabla_{\|} \mathcal{N}}{\mathcal{N}},
$$

where $\nabla_{\|} \tilde{n} / \tilde{n}$ follows from the zeroth-order moment of (A.1) in the absence of ICRF heating, i.e. $\mathcal{N}=1$ and thus $\nabla \mathcal{N}=0$.

On the HFS, it holds $\mathcal{N}=\mathcal{T}_{-}=T_{\perp} / T_{\perp \text {,ic }}$ and (A.2) reduces to equation (9) in [6],

$$
\frac{\nabla_{\|} n}{n}=-q \frac{\nabla_{\|} \Phi}{T_{\|}}+\frac{\nabla_{\|}\left(m V^{2}\right)}{2 T_{\|}}+\frac{\nabla_{\|} T_{\perp}}{T_{\perp}} .
$$


[1] Ingesson, L. C., Chen, H., Helander, P., and MAntsinen, M. J., Plasma Physics and Controlled Fusion 42 (2000) 161.

[2] REINKE, M. L., HUTCHINSON, I. H., RICE, J. E., et al., Plasma Physics and Controlled Fusion 54 (2012) 045004.

[3] CASSOn, F. J., ANGIOni, C., BELli, E. A., et al., Plasma Physics and Controlled Fusion 57 (2015) 014031.

[4] CHOE, W., Chang, C. S., and ONO, M., Physics of Plasmas 2 (1995) 2044.

[5] HINTON, F. L. and WONG, S. K., Physics of Fluids 28 (1985) 3082.

[6] BILATO, R., MAJ, O., and ANGIONI, C., Nuclear Fusion 54 (2014) 072003.

[7] KIPTILY, V., ADAMS, J., BERTALOT, L., et al., Nuclear Fusion 45 (2005) L21.

[8] DENDY, R. O., HASTIE, R. J., MCCLEMENTS, K. G., and MARTIN, T. J., Physics of Plasmas 2 (1995) 1623.

[9] BRAmbiLla, M., Kinetic Theory of Plasma Waves, Oxford University Press, USA, 1998.

[10] KERBEL, G. D. and MCCOY, M. G., Physics of Fluids 28 (1985) 3629.

[11] COOPER, W., GRAVES, J., HIRSHMAN, S., et al., Nuclear Fusion 46 (2006) 683.

[12] JUCKER, M., GRAVES, J., COOPER, W., et al., Computer Physics Communications 182 (2011) 912 .

[13] KAZAKOV, Y. O., PUSZTAI, I., FÜLÖP, T., and JOHNSON, T., Plasma Physics and Controlled Fusion 54 (2012) 105010.

[14] STIX, T., Nuclear Fusion 15 (1975) 737.

[15] JAEGER, E. F., BERRY, L. A., AHERN, S. D., et al., Physics of Plasmas 13 (2006) 056101.

[16] GRAVES, J. P., COOPER, W. A., CODA, S., and ERIKSSON, L. C., AIP Conference Proceedings 871 (2006) 350.

[17] WESSON, J., Nuclear Fusion 37 (1997) 577.

[18] HAMMETT, G. W., Fast ion studies of ion cyclotron heating in the PLT tokamak, PhD thesis, Princeton University, 1986.

[19] DE VRIES, P. C., RANTAMKI, K. M., GIROUD, C., et al., Plasma Physics and Controlled Fusion 48 (2006) 1693.

[20] POlitzer, P., PETTy, C., JAYAKumaR, R., et al., Nuclear Fusion 48 (2008) 075001.

[21] MCDERMOTT, R. M., ANGIONI, C., DUX, R., et al., Plasma Physics and Controlled Fusion 53 (2011) 124013.

[22] Angioni, C., MCDermott, R. M., CASSON, F. J., et al., Phys. Rev. Lett. 107 (2011) 215003.

[23] Angioni, C., MANTiCA, P., PTteriCH, T., et al., Nuclear Fusion 54 (2014) 083028. 